\title{
Long-term protective effects of the angiotensin receptor blocker telmisartan on epirubicin-induced inflammation, oxidative stress and myocardial dysfunction
}

\author{
MARIELE DESSI $^{1 *}$, ALESSANDRA PIRAS ${ }^{2 *}$, CLELIA MADEDDU $^{1}$, CHRISTIAN CADEDDU $^{2}$, MARTINO DEIDDA $^{2}$, \\ ELENA MASSA $^{1}$, GIORGIA ANTONI ${ }^{1}$, GIOVANNI MANTOVANI ${ }^{1}$ and GIUSEPPE MERCURO ${ }^{2}$ \\ Departments of ${ }^{1}$ Medical Oncology, and ${ }^{2}$ Cardiovascular and Neurological Sciences, \\ University of Cagliari, 09042 Monserrato (CA), Italy
}

Received April 13, 2011; Accepted June 17, 2011

DOI: $10.3892 /$ etm.2011.305

\begin{abstract}
Chronic inflammation, oxidative stress and the renin-angiotensin system (RAS) play a significant role in chemotherapy-induced cardiotoxicity (CTX). Telmisartan (TEL), an antagonist of the angiotensin II type-1 receptor, was found to reduce anthracycline (ANT)-induced CTX. We carried out a phase II placebo (PLA)-controlled randomized trial to assess the possible role of TEL in the prevention of cardiac subclinical damage induced by epirubicin (EPI). Forty-nine patients (mean age \pm SD, 53.0 \pm 8 years), cardiovascular diseasefree with cancer at different sites and eligible for EPI-based treatment, were randomized to one of two arms: TEL n=25; PLA $\mathrm{n}=24$. A conventional echocardiography equipped with Tissue
\end{abstract}

Correspondence to: Professor Giovanni Mantovani, Cattedra di Oncologia Medica, Università di Cagliari, Azienda OspedalieroUniversitaria di Cagliari, S.S. 554, Km 4,500, 09042 Monserrato (CA), Italy

E-mail: mantovan@medicina.unica.it

*Contributed equally

Abbreviations: ANTs, Anthracyclines; CTX, chemotherapyinduced cardiotoxicity; IL-6, interleukin-6; TNF, tumor necrosis factor; ROS, reactive oxygen species; EPI, epirubicin; FU, follow-up; RAS, renin-angiotensin system; AT1Rs, angiotensin II type-1 receptors; TEL, telmisartan; EGF, epidermal growth factor; PPAR, proliferator-activated receptor; PLA, placebo; SR, strain rate; LVEF, left ventricle ejection fraction; ECOG, Eastern Cooperative Oncology Group; TDI, Tissue Doppler Imaging; GPx, glutathione peroxidase; SOD, superoxide dismutase; S, strain; LVEDD, left ventricular end diastolic diameter; PWD, pulsed wave Doppler; E, early diastolic peak velocities; A, late diastolic peak velocities; DecT, deceleration time; IVS, interventricular septum; Sm, systole; IVRT, isovolumic relaxation time; Em, early diastole; Am, late diastole; FORT, free oxygen radical test; PD, disease progression;

Key words: epirubicin-induced cardiotoxicity, cytokines, oxidative stress, renin-angiotensin system, telmisartan
Doppler imaging, strain and strain rate (SR) was performed, and serum levels of proinflammatory cytokines, IL-6 and TNF- $\alpha$, and oxidative stress parameters, reactive oxygen species (ROS) and glutathione peroxidase were determined. All assessments were carried out at baseline, after every $100 \mathrm{mg} / \mathrm{m}^{2}$ of EPI dose and at the 12-month follow-up (FU). A significant reduction in the SR peak both in the TEL and PLA arms was observed at $\mathrm{t}_{2}$ (cumulative dose of $200 \mathrm{mg} / \mathrm{m}^{2}$ of EPI) in comparison to $\mathrm{t}_{0}$. Conversely, at $\mathrm{t}_{3}\left(300 \mathrm{mg} / \mathrm{m}^{2} \mathrm{EPI}\right), \mathrm{t}_{4}\left(400 \mathrm{mg} / \mathrm{m}^{2} \mathrm{EPI}\right)$ and the 12-month FU, the SR increased reaching the normal range only in the TEL arm, while in the PLA arm the SR remained significantly lower as compared to $\mathrm{t}_{0}$ (baseline). The differences between SR changes in the PLA and TEL arms were significant from $300 \mathrm{mg} / \mathrm{m}^{2}$ EPI $\left(\mathrm{t}_{3}\right)$ up to the 12-month FU. Serum levels of IL-6 increased significantly in the PLA arm at $200 \mathrm{mg} / \mathrm{m}^{2}$ EPI $\left(t_{2}\right)$ in comparison to baseline, but remained unchanged in the TEL arm. The same trend was demonstrated for ROS levels which significantly increased at $t_{2}$ vs. baseline in the PLA arm, while remained unchanged in the TEL arm. The mean change in ROS and IL-6 at $t_{2}$ was significantly different between the two arms. In the present study, we confirmed at the 3-month FU a trend toward a decrease in ROS and IL- 6 from $t_{2}$ in the PLA arm. Our results suggest that TEL is able to reverse acute (early) EPI-induced myocardial dysfunction and to maintain later a normal systolic function up to the 12-month FU. These effects are likely to be due to different mechanisms, RAS blockade and prevention of chronic inflammation/oxidative stress.

\section{Introduction}

Anthracyclines (ANTs) are among the most effective anticancer drugs used in the treatment of a wide spectrum of malignancies. Regrettably, their clinical use is limited by the occurrence of dose-related cardiotoxicity (4).

Several studies have shown that chemotherapy-induced cardiotoxicity (CTX) produced by ANTs is at least partially mediated by chronic inflammation and oxidative stress, with proinflammatory cytokines, interleukin-6 (IL-6) and tumor necrosis factor (TNF)- $\alpha$ and reactive oxygen species (ROS) (2) playing a central role $(5,6)$. It has also been shown that the use of a conventional cardioprotective agent, such as dexrazoxane, 
together with chemotherapy, reduces the expression of the NRF-2 gene (responsible for oxidative stress response), which is overexpressed in patients receiving ANT alone (2).

We previously identified an epirubicin (EPI)-induced early myocardial dysfunction, detected after a low dose $(200 \mathrm{mg} /$ $\mathrm{m}^{2}$ ) of EPI (7). This dysfunction was shown to be correlated with a significant increase in several biological markers of inflammation and oxidative stress, and persisted throughout EPI treatment and up to the 18-month follow-up (FU) (8).

Recent accumulating evidence suggests the involvement of the renin-angiotensin system (RAS) in ANT-induced CTX. Angiotensin II plays a crucial role, not only as a vasoconstrictor agent, but also as a mitogenic factor by interacting with angiotensin II type-1 receptors (AT1Rs) in cardiovascular myocytes (9). Cardiac dysfunction after doxorubicin has not been demonstrated in the knockout rat for the AT1R gene, a finding confirmed by the absence of apoptosis and myofibrillar damage (10). In a recent study, the cardioprotective effect of the angiotensin receptor blocker (11), telmisartan (TEL), has been demonstrated in rats exposed to ANT (12). The authors argued that the effect was sustained through a decrease in oxidative stress, which in turn was able to reduce the structural damage of cardiomyocytes. Regarding the possible role of ARBs in mitogenesis and angiogenesis, these drugs were found to suppress the signal transduction mediated by growth factors, such as the epidermal growth factor (EGF), through AT1R antagonism (13). Furthermore, the ARB, telmisartan, was shown to inhibit the proliferation of prostate cancer cells through activation of the peroxisome proliferator-activated receptor (PPAR)- $\gamma$ (3).

In a previous phase II placebo (PLA)-controlled study we used TEL in order to prevent EPI-induced myocardial damage (14). We aimed to exploit the ability of this drug to inhibit the production of superoxide radicals by mitochondrial NADPHdependent oxidase and xanthine oxidase (15), and to, at least partially, antagonize PPAR- $\gamma$ activation (16). In this earlier study, we reported that TEL was able to reduce EPI-induced oxidative stress/chronic inflammation and to reverse early myocardial impairment (14).

The aim of the present study was to confirm a long-lasting cardioprotective activity of TEL, up to a 12-month FU (3), in preserving the systolic function [assessed as strain rate (SR)] (primary endpoint) and reducing inflammation and oxidative stress (secondary endpoint) in a population of cancer patients treated with EPI. We also assessed the changes in the above parameters at intermediate time points (3- and 6-month FU) as tertiary endpoints.

\section{Patients and methods}

Patient population and study protocol. From September 2008 to October 2009, we enrolled 49 consecutive eligible patients (male/female ratio, 12/37) with histologically confirmed tumors at different sites, previously untreated. All eligible patients were included. The majority of patients $(n=30)$ had early-stage cancer and only a minority had oligometastatic disease. Patients were eligible for an EPI-based chemotherapy regimen and were followed up until a maximal cumulative dose of $400 \pm 20 \mathrm{mg} / \mathrm{m}^{2}$ (mean $\pm \mathrm{SD}$ ), according to the international standardized protocols for EPI-based administration. Inclusion
Table I. Clinical characteristics of the patients.

\begin{tabular}{lcc}
\hline & PLA & TEL \\
\hline Patients & 24 & 25 \\
Gender (male/female) & $6 / 18$ & $6 / 19$ \\
Age, years (mean \pm SD) & $53 \pm 10$ & $52.9 \pm 9$ \\
Tumor site & & \\
Endometrium & 9 & 12 \\
Salivary gland & 1 & 0 \\
Non Hodgkin's lymphoma & 2 & 1 \\
Breast & 10 & 8 \\
Ovary & 1 & 4 \\
Lung (NSCLC) & 1 & 0 \\
Stage & & \\
I & 13 & 14 \\
II & 7 & 6 \\
III & 3 & 4 \\
IV & 1 & 1 \\
ECOG PS & & \\
0 & 15 & 18 \\
1 & 4 & 6 \\
2 & 5 & 1 \\
BMI & & \\
$<18.5$ & 0 & 0 \\
$18.5-25$ & 20 & 5 \\
$>25$ & 4 & \\
\hline
\end{tabular}

BMI, body mass index; ECOG PS, Eastern Cooperative Oncology Group performance status; NSCLC, non-small cell lung cancer; PLA, placebo arm; TEL, telmisartan arm.

criteria were the following: patients 18-70 years of age; an echocardiographic left ventricle ejection fraction (LVEF) value $\geq 55 \%$; an $S R$ value in the normal range $(1.7-2.1 \mathrm{~cm} /$ sec); an Eastern Cooperative Oncology Group (ECOG) performance status score of 0-2 (11); normal hepatic and renal function (bilirubin $\leq 1.5 \mathrm{mg} / \mathrm{dl}$ and creatinine $\leq 2.0 \mathrm{mg} / \mathrm{dl}$ ); no concomitant medications known to interfere with inflammatory and oxidative stress parameters. Patients were not eligible if they had a history of cardiac disease, hypertension, diabetes and/or had been previously treated with mediastinal radiotherapy. The study was a one-institution 'independent', randomized, PLA-controlled trial and was approved by the Institutional Ethics Committee of the "Azienda Ospedaliero Universitaria' of Cagliari, Italy. Written informed consent was obtained by all patients included in the study.

A blind randomization was performed: 25 patients were randomized by a block randomization technique to the TEL (1) arm and 24 to the PLA arm. Clinical characteristics of patients in each arm are summarized in Table I. Patients were treated with $40 \mathrm{mg}$ of TEL (Micardis ${ }^{\circledR}$, Boehringer-Ingelheim, Milan, Italy), 1 tablet/day, or PLA starting 1 week before the beginning of EPI treatment and up to 6 months after EPI discontinuation. The PLA tablets were supplied from the insitutional pharmacy and were identical in appearance and taste to TEL. The consort diagram is shown in Fig. 1. 


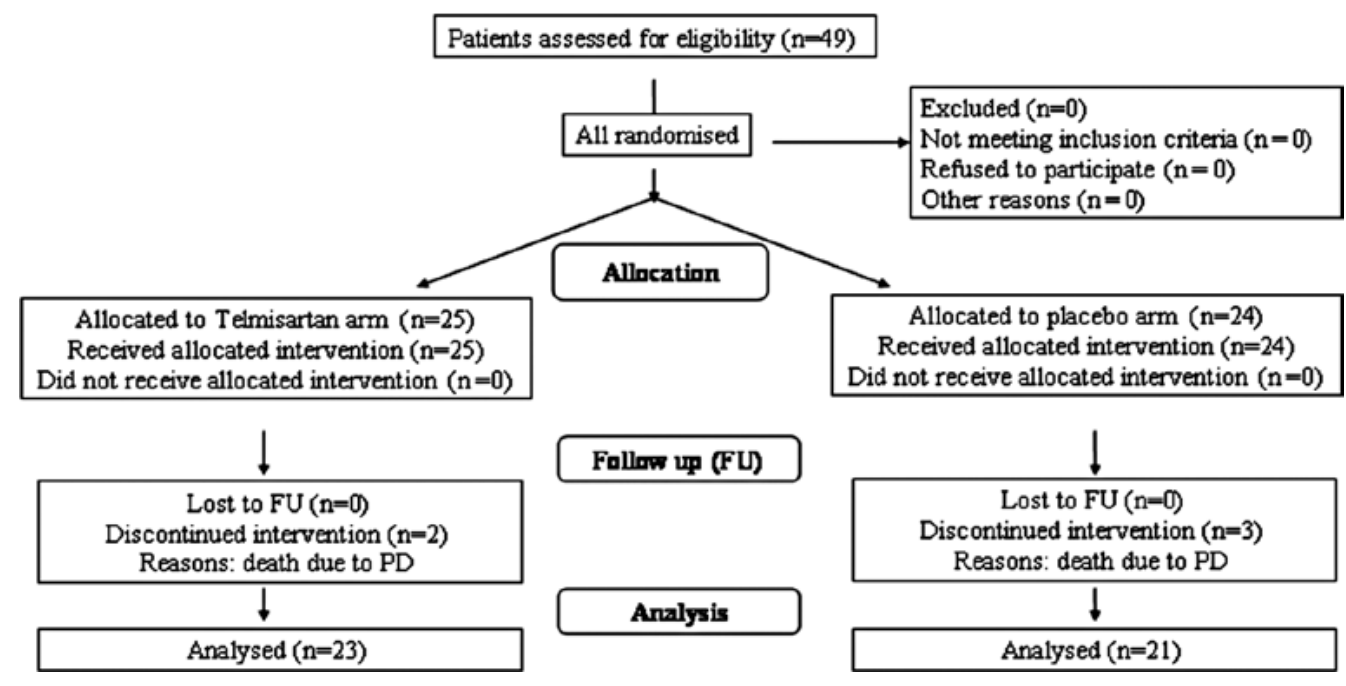

Figure 1. Consort diagram.

Clinical and laboratory assessments. At enrollment, before randomization as well as after each subsequent administration of EPI, patients underwent a physical examination, blood pressure measurement, 12-lead electrocardiogram and echocardiographic analysis (conventional and Tissue Doppler Imaging; TDI). The following laboratory tests were carried out: blood and platelet count, BUN, uric acid, creatinine, blood and urine electrolytes, direct and indirect bilirubin, AST, ALT, $\gamma \mathrm{GT}$, alkaline phosphatase, iron, ferritinemia and blood transferrin. In all patients, blood samples were collected for the assessment of circulating levels of proinflammatory cytokines (IL-6 and TNF- $\alpha$ ), ROS and antioxidant enzymes, glutathione peroxidase (GPx) and superoxide dismutase (SOD). The instrumental and laboratory variables were assessed at baseline $\left(\mathrm{t}_{0}\right), 7$ days after reaching the EPI dose of 100, 200, 300 and $400 \mathrm{mg} / \mathrm{m}^{2}\left(\mathrm{t}_{1}, \mathrm{t}_{2}, \mathrm{t}_{3}\right.$ and $t_{4}$, respectively) and at the 3,6 and 12-month FU (3). The reported doses of EPI were always intended to be cumulative.

Conventional echocardiography and TDI. Echocardiographic images were recorded using a commercially available system equipped with TDI, strain (S) and strain rate (SR) imaging (Toshiba APLIO CV ultrasound system-SSA 770A/CV; Toshiba Corp., Tochigi, Japan). LVEF was obtained from the apical 4- and 2-chamber views according to Simpson's rule and was considered abnormal under $55 \%$.

Conventional echocardiography parameters, such as left ventricular end diastolic diameter (LVEDD) and atrial dimensions, were assessed in both arms.

A pulsed wave Doppler (PWD) examination of the LV inflow from the 4-chamber view was performed, with the sample volume placed between the mitral leaflet tips and the early (E) and late (A) diastolic peak velocities; E deceleration time (DecT) was measured and the E/A ratio was derived. We evaluated the longitudinal function using pulsed TDI at the mitral annulus, placing the sample volume in the basal segment of the interventricular septum (IVS) from the apical 4-chamber view; peak velocities in systole $\left(\mathrm{S}_{\mathrm{m}}\right)$, isovolumic relaxation time (IVRT), early $\left(\mathrm{E}_{\mathrm{m}}\right)$ and late $\left(\mathrm{A}_{\mathrm{m}}\right)$ diastole were measured. LV longitudinal function was evaluated from raw data; myocardial S and SR were also quantified in the IVS. The same experienced echocardiographer carried out all examinations of each individual patient. To reduce interobserver variability, all echocardiographic data were randomly read by a second experienced observer and an average value for each measurement was calculated. Reproducibility of TDI parameters in our laboratory have been previously documented.

Inflammatory and oxidative stress markers. Blood samples were obtained from all patients by venipuncture of the antecubital vein at 8 a.m., after overnight fasting. Levels of IL-6 and TNF- $\alpha$ were determined by enzyme-linked immunosorbent assay (Immunotech, Marseille, France) and expressed in pg/ $\mathrm{ml}$. Blood levels of ROS were determined using fresh heparinized blood samples using the free oxygen radical test (FORT). Results are expressed as FORT units (U), where 1 FORT U corresponds to $0.26 \mathrm{mg} / \mathrm{l}$ of $\mathrm{H}_{2} \mathrm{O}_{2}$. The erythrocyte antioxidant enzymes, GPx and SOD, were measured by a photometer using a commercially available kit (Ransod; Randox Laboratory, Crumlin, UK) and expressed as $\mathrm{U} / \mathrm{l}$ and $\mathrm{U} / \mathrm{ml}$, respectively.

Statistical analysis. Considering an $\alpha$ type error of 0.05 , a $\beta$ type error of 0.10 and a difference in SR changes between arms of $10 \%$ of the primary endpoint (SR change) as clinically meaningful, 50 patients were enrolled in each arm. An interim analysis on the basis of the early-stopping rules was planned after the enrollment of 50 patients. Treatment arms were compared by the Student's t-test for changes. Differences between values measured at different times (different EPI doses) and at the 3-, 6- and 12-month FU were calculated by the ANOVA test. The correlation between instrumental (TDI) and laboratory variables was assessed by Pearson's t-test (or Spearman's t-test for non-parametric variables). Significant relationships were then examined by multivariate linear regression analysis. Results were considered significant for $\mathrm{p}$-values $\leq 0.05$. Data are reported as the means \pm SD. Statistical analysis was performed using SPSS version 14 for Windows.

\section{Results}

The two treatment arms were well-matched in terms of age, ECOG performance status, site and stage of disease at enroll- 
Table II. Conventional echocardiographic parameters of systolic and diastolic function in both arms.

\begin{tabular}{|c|c|c|c|c|c|}
\hline Conventional echo & $\mathrm{t}_{0}(\mathrm{n}=49)$ & $t_{2}(n=49)$ & $t_{3}(n=49)$ & $t_{4}(n=49)$ & 12-month FU $(n=44)$ \\
\hline \multicolumn{6}{|l|}{ LVEF } \\
\hline PLA & $66 \pm 5 \%$ & $68 \pm 6 \%$ & $66 \pm 5 \%$ & $66 \pm 5 \%$ & $67 \pm 5 \%$ \\
\hline TEL & $66 \pm 7 \%$ & $67 \pm 6 \%$ & $68 \pm 4 \%$ & $70 \pm 6 \%$ & $68 \pm 4 \%$ \\
\hline \multicolumn{6}{|l|}{ DecT } \\
\hline PLA & $0.22 \pm 0.04$ & $0.24 \pm 0.05$ & $0.22 \pm 0.02$ & $0.23 \pm 0.04$ & $0.22 \pm 0.03$ \\
\hline TEL & $0.19 \pm 0.04$ & $0.21 \pm 0.04$ & $0.20 \pm 0.02$ & $0.21 \pm 0.03$ & $0.21 \pm 0.04$ \\
\hline \multicolumn{6}{|l|}{$\mathrm{E} / \mathrm{A}$} \\
\hline PLA & $1.13 \pm 0.14$ & $1.08 \pm 0.12$ & $0.92 \pm 0.05^{\mathrm{a}}$ & $0.90 \pm 0.06^{\mathrm{a}}$ & $1.06 \pm 0.42$ \\
\hline TEL & $0.96 \pm 0.12$ & $0.86 \pm 0.08$ & $0.83 \pm 0.07$ & $0.95 \pm 0.14$ & $0.87 \pm 0.31$ \\
\hline
\end{tabular}

LVEF, left ventricle ejection fraction; DecT, deceleration time; E/A, early and late diastolic peak velocity ratio; $\mathrm{t}_{0}$, baseline; $\mathrm{t}_{2}, 200 \mathrm{mg} / \mathrm{m}^{2} \mathrm{EPI}$; $\mathrm{t}_{3}, 300 \mathrm{mg} / \mathrm{m}^{2} \mathrm{EPI} ; \mathrm{t}_{4}, 400 \mathrm{mg} / \mathrm{m}^{2} \mathrm{EPI} ;{ }^{\mathrm{a}} \mathrm{p}<0.05$ vs. $\mathrm{t}_{0}$.

Table III. TDI echocardiographic parameters of systolic and diastolic function in both arms.

\begin{tabular}{|c|c|c|c|c|c|}
\hline TDI echo & $t_{0}(n=49)$ & $t_{2}(n=49)$ & $t_{3}(n=49)$ & $t_{4}(n=49)$ & 12-month FU $(n=44)$ \\
\hline \multicolumn{6}{|l|}{$\mathrm{E}_{\mathrm{m}}$} \\
\hline PLA & $8.66 \pm 4.90$ & $8.64 \pm 6.07$ & $7.73 \pm 4.90$ & $7.54 \pm 3.50$ & $7.88 \pm 2.07$ \\
\hline TEL & $7.89 \pm 2.14$ & $7.33 \pm 2.45$ & $7.53 \pm 2.17$ & $6.93 \pm 1.46$ & $7.74 \pm 1.58$ \\
\hline \multicolumn{6}{|l|}{$\mathrm{E}_{\mathrm{m}} / \mathrm{A}_{\mathrm{m}}$} \\
\hline PLA & $1.13 \pm 0.26$ & $0.85 \pm 0.35^{\mathrm{a}}$ & $0.72 \pm 0.30^{\mathrm{a}}$ & $0.75 \pm 0.32^{\mathrm{a}}$ & $0.96 \pm 0.27$ \\
\hline TEL & $0.90 \pm 0.11$ & $0.84 \pm 0.07$ & $0.85 \pm 0.05$ & $0.71 \pm 0.23$ & $0.78 \pm 0.28$ \\
\hline \multicolumn{6}{|l|}{$S_{m}$} \\
\hline PLA & $7.15 \pm 0.65$ & $7.28 \pm 1.71$ & $7.28 \pm 0.82$ & $6.83 \pm 0.69$ & $7.25 \pm 1.37$ \\
\hline TEL & $7.33 \pm 1.76$ & $7.09 \pm 1.21$ & $7.04 \pm 1.12$ & $7.29 \pm 1.08$ & $7.33 \pm 1.22$ \\
\hline \multicolumn{6}{|l|}{ Strain } \\
\hline PLA & $20.89 \pm 1.96$ & $20.75 \pm 2.06$ & $18.00 \pm 2.55$ & $18.65 \pm 1.25$ & $16.78 \pm 2.22$ \\
\hline TEL & $22.80 \pm 1.54$ & $21.20 \pm 1.86$ & $20.40 \pm 0.94$ & $19.90 \pm 0.92$ & $19.64 \pm 1.82$ \\
\hline
\end{tabular}

$\mathrm{E}_{\mathrm{m}}$, TD early diastolic peak velocity; $\mathrm{E}_{\mathrm{m}} / \mathrm{A}_{\mathrm{m}}$, TD early and late diastolic peak velocity ratio; $\mathrm{S}_{\mathrm{m}}$, TD systolic peak velocity. ${ }^{\mathrm{a}} \mathrm{p}<0.05 \mathrm{vs} . \mathrm{t}_{0}$.

ment (Table I). All patients reached the scheduled cumulative EPI dose of $400 \mathrm{mg} / \mathrm{m}^{2}$. Regarding tumor history, it should be noted that overall 5 patients died at $5 \pm 2$ months after the end of EPI treatment due to disease progression (PD): 2 patients in the TEL arm and 3 patients in the PLA arm (Fig. 1). Moreover, at the 12 -month FU, PD was noted in $1 / 23$ patients in the TEL arm and in $2 / 21$ patients in the PLA arm.

ECG monitoring. At ECG monitoring, a normal morphology was observed throughout the treatment in 33 patients; in 16 patients (9 in the TEL and 7 in the PLA arm) widespread and unspecified changes were observed during the ventricular repolarization phase concurrent with $t_{2}$, with no significant differences between the TEL and PLA arm.

Conventional echocardiography and TDI. In the PLA arm, 1 week after $t_{2}$, as well as at $t_{3}$, a significant LV diastolic impairment was observed, represented by a reduction in the E/A ratio at PWD examination $(\mathrm{p}<0.05$; Table II). In the TEL arm, a slight reduction in the E/A ratio was observed, which, however, did not reach statistical significance. No significant abnormalities of LVEF and DecT were found in any of the two arms throughout the treatment (Table II).

Conventional echocardiography parameters, LVEDD and atrial dimensions, were in the normal range and did not differ between arms at baseline; they did not change during treatment up to the 12-month FU (data not shown).

TDI echocardiographic analysis revealed in the PLA arm an LV diastolic impairment, indicated by a reduction in the $\mathrm{E}_{\mathrm{m}} /$ $A_{m}$ ratio measured in the basal portion of IVS, first recognized at $t_{2}(p<0.05$; Table III). This impaired function persisted throughout the treatment, at $t_{3}(p<0.05)$ and $t_{4}(p<0.05$; Table III), whereas in the TEL arm this diastolic impairment did not occur (the $\mathrm{E}_{\mathrm{m}} / \mathrm{A}_{\mathrm{m}}$ ratio did not change significantly). At the 12-month $\mathrm{FU}$, the $\mathrm{E}_{\mathrm{m}} / \mathrm{A}_{\mathrm{m}}$ ratio returned within the $\mathrm{t}_{0}$ range in the PLA arm. The other TDI parameters $\left(E_{m}, S_{m}\right.$ and $\left.S\right)$ did not show any significant changes during treatment up to the 12-month FU in any of the two arms (Table III).

A significant reduction in the SR peak both in the TEL and PLA arms was observed at $t_{2}$ (cumulative dose of $200 \mathrm{mg}$ / 


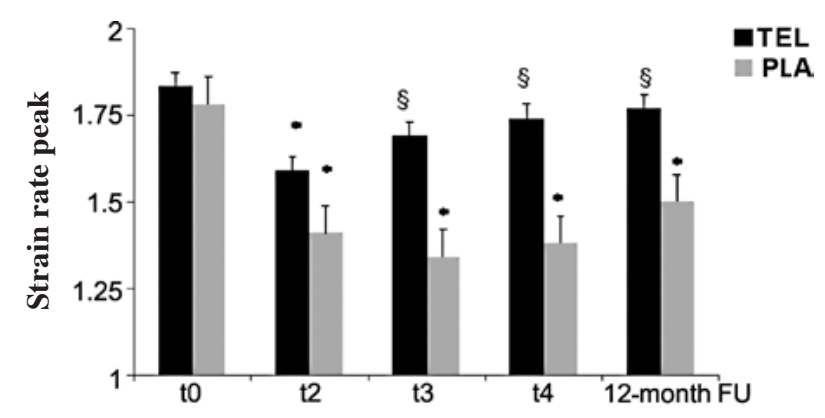

Figure 2. SR analysis with TDI in the two arms. ${ }^{*} \mathrm{p}<0,05$ vs. baseline; ${ }^{s} \mathrm{p}<0.05$ between arms.

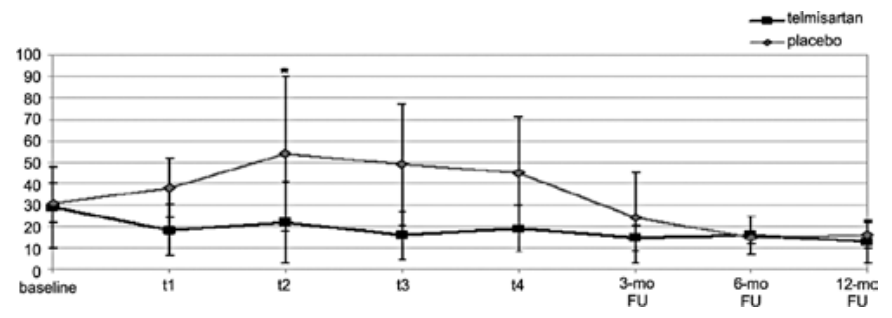

Figure 3. Serum levels of IL-6 $(\mathrm{pg} / \mathrm{ml})$ during the EPI treatment and FU in the TEL and PLA arms. " $p<0.05$ between arms.

$\left.\mathrm{m}^{2} \mathrm{EPI}\right)$ in comparison to $\mathrm{t}_{0}(1.45 \pm 0.33$ vs. $1.54 \pm 0.35 \mathrm{~s}-1$; NS $)$. Conversely, at $\mathrm{t}_{3}\left(300 \mathrm{mg} / \mathrm{m}^{2} \mathrm{EPI}\right), \mathrm{t}_{4}\left(400 \mathrm{mg} / \mathrm{m}^{2} \mathrm{EPI}\right)$ and the 12-month FU, the SR increased reaching the normal range only in the TEL arm, while in the PLA arm the SR remained significantly lower as compared to $\mathrm{t}_{0}$ (baseline).

The differences between SR changes in the PLA and TEL arms were significant at $\mathrm{t}_{3}, \mathrm{t}_{4}$ and the 12-month FU (Fig. 2).

Inflammation and oxidative stress markers. Serum levels of IL-6 increased significantly in the PLA arm at $\mathrm{t}_{2}$ in comparison to baseline $(68.8 \pm 52.6$ vs. $31 \pm 8.8 \mathrm{pg} / \mathrm{ml}, \mathrm{p}<0.005)$, but remained unchanged in the TEL arm ( $28 \pm 18.1$ vs. $26 \pm 18.8 \mathrm{pg} /$ ml) (Fig. 3). The same trend was shown for ROS levels which significantly increased at $t_{2}$ vs. baseline in the PLA arm ( $518 \pm 65$ vs. $407.3 \pm 126$ FORT-U, p $<0.05$ ), while they remained unchanged in the TEL arm $(438 \pm 105$ vs. $452 \pm 61$ FORT-U; NS)

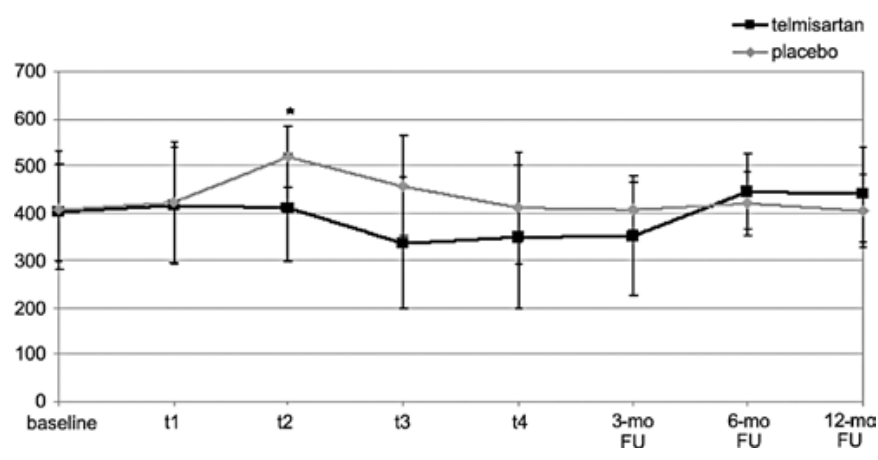

Figure 4. Blood levels of ROS (FORT U) during the EPI treatment and FU in the TEL and PLA arms. ${ }^{*} \mathrm{p}<0.05$ between arms.

(Fig. 4). The mean change in IL-6 and ROS at $\mathrm{t}_{2}$ was significantly different between the two arms (Figs. 3 and 4).

The other laboratory parameters (TNF- $\alpha$, GPx and SOD) did not show significant changes in any of the two arms throughout the study (Table IV).

Correlations between echocardiographic and laboratory data. In the PLA arm, the early impairment of the TDI parameters (calculated as $\Delta$ by subtracting the values at $\mathrm{t}_{2}$ from the baseline values) was also correlated with changes in circulating levels of inflammation and oxidative stress markers. Significant correlations were found between $\triangle \mathrm{SR}$, increase in IL-6 and ROS ( $r=0.58, p<0.005$ and $r=0.51, p<0.05$, respectively) in the PLA arm.

Safety. In regards to TEL, the drug was well tolerated by all patients throughout the study. However, a significant hypotension episode (blood pressure values $<95 / 50 \mathrm{mmHg}$ ) was observed only once in 2 patients (approximately corresponding to $t_{2}$ and $t_{3}$ ). For this event, the TEL dose was reduced from 40 to $20 \mathrm{mg} / \mathrm{day}$ for 2 subsequent weeks, and thereafter the full dose of $40 \mathrm{mg}$ /day was re-established.

As for EPI-related side effects, the only significant adverse event was a grade $3 / 4$ neutropenia observed at $t_{3}$ in 6 patients who needed administration of granulocyte colony-stimulating factor and a postponement of the subsequent EPI-based cycle.

Table IV. Inflammation and oxidative stress markers in both arms.

\begin{tabular}{lcccccc}
\hline Parameters & $\mathrm{t}_{0}(\mathrm{n}=49)$ & $\mathrm{t}_{2}(\mathrm{n}=49)$ & $\mathrm{t}_{3}(\mathrm{n}=49)$ & $\mathrm{t}_{4}(\mathrm{n}=49)$ & 12-month FU $(\mathrm{n}=44)$ & $\mathrm{p}$-value (ANOVA test) \\
\hline TNF- $\alpha$ & & & & & & \\
PLA & $30.1 \pm 9.0$ & $47.1 \pm 4.6$ & $30.0 \pm 14.4$ & $25.8 \pm 8.6$ & $30.0 \pm 14.4$ & 0.32 \\
TEL & $23.5 \pm 5.5$ & $22.3 \pm 10.6$ & $35.1 \pm 8.6$ & $25.9 \pm 4.2$ & $35.1 \pm 8.6$ & 0.56 \\
GPx & & & & & & \\
PLA & $7,386 \pm 3,041$ & $6,898 \pm 1,552$ & $9,427 \pm 3,078$ & $10,232 \pm 1,875$ & $9,427 \pm 3,078$ & 0.56 \\
TEL & $7,415 \pm 2,182$ & $6,381 \pm 2,137$ & $7,263 \pm 2,998$ & $7,479 \pm 1,769$ & $7,263 \pm 2,998$ & 0.88 \\
SOD & & & & & & \\
PLA & $130.0 \pm 9.2$ & $138.0 \pm 2.1$ & $193.0 \pm 41.0$ & $148.0 \pm 38.0$ & $135.0 \pm 26.0$ & 0.32 \\
TEL & $126.1 \pm 41.6$ & $150.0 \pm 37.0$ & $140.0 \pm 40.0$ & $145.0 \pm 74.0$ & $150.0 \pm 37.0$ & 0.95 \\
\hline
\end{tabular}

TNF, tumor necrosis factor; GPx, glutathione peroxidase; SOD, superoxide dismutase. 


\section{Discussion}

The present study confirmed the results reported in our previous work (14) and various important novel findings were demonstrated: i) a reduction in the SR peak at $\mathrm{t}_{2}$ in both treatment arms with no statistical difference between the two arms; ii) a persistent reduction in the SR peak in the PLA arm at $t_{3}$ and $t_{4}$ and, more importantly, the SR peak reduction persisted also at the 12-month FU; iii) a recovery of the SR peak in the TEL arm which, starting from $t_{3}$, reached values within the range of $\mathrm{t}_{0}$ which were persistent up to the 12-month $\mathrm{FU}$ (data shown for the first time in the present study); iv) serum levels of IL-6 increased significantly in the PLA arm from $t_{0}$ to $t_{2}$, then decreased subsequently to the baseline range up to the 12-month FU (the latter is a new finding), whereas serum levels of IL- 6 in the TEL arm remained unchanged from $t_{0}$ to the 12-month FU; v) blood levels of ROS demonstrated a superimposable pattern to that of IL-6; and vi) changes in the SR peak, an echocardiographic equivalent of early myocardial systolic dysfunction revealed by TDI, correlated with changes in the levels of IL-6 and ROS, which are indicative of the body inflammatory and oxidative stress status, only in the PLA arm at $t_{2}$. The results of the present study prompted us to discontinue the study at the planned interim analysis based on the early-stopping rules for superiority of the TEL arm due mainly to ethical reasons.

The findings of the present study confirmed that AT1R blockade by TEL, administered 1 week before and throughout the duration of EPI treatment, was able to initially $\left(\mathrm{t}_{2}\right)$ reduce and later $\left(t_{3}\right.$ and $\left.t_{4}\right)$ reverse EPI-induced cardiac abnormalities. This effect was long-lasting and still persisted at the 12-month FU. Moreover, TEL co-administration also prevented increases in IL-6 and ROS levels after EPI administration.

In previous reports, we found that a measurable decline in the SR peak, currently regarded as the earliest sign of subclinical CTX, may be detected in EPI-treated patients long before the clinical evidence of heart failure (7). The subtle systolic impairment appeared after $200 \mathrm{mg} / \mathrm{m}^{2}$ EPI, a dose which was, until recently, considered insufficient to induce cardiac injury (17). Moreover, a progressive EPI-induced myocardial dysfunction, which was present even at the 18-month FU in a population of patients not treated with the cardioprotective drugs (8), was not noted in the present study in the TEL arm. As reported above, TEL was shown to reverse the early myocardial dysfunction observed at $200 \mathrm{mg} / \mathrm{m}^{2}$ EPI and, importantly, its beneficial cardioprotective effect persisted up to the 12-month FU, i.e., 12 months after discontinuation of EPI chemotherapy and 6 months after the end of TEL coverage.

A large body of evidence has confirmed the role of the AT1Rs in mediating the damage caused by from myocardial ischemia/reperfusion, resulting from acute ANT-induced CTX $(18,19)$. Accordingly, ANTs were found to induce myofibrillar loss, increase the number of apoptotic cells and significantly impair cardiac function in control mice, but not in AT1Rknockout mice or in animals treated with an AT1R antagonist (9). This evidence suggests that an ARB, such as TEL, may be able to prevent ANT-induced CTX.

In a recent study, a protective effect of TEL against acute ANT-induced CTX was demonstrated in rats; it was shown that pre-treatment with the ARB TEL elicited a normalization of significant biochemical parameters and reduced cardiac tissue damage (12).

Therefore, the present study supports the previously reported role of the RAS in the pathophysiology of chemotherapy-induced CTX; in particular, it also demonstrates for the first time in, a clinical trial, the anti-inflammatory and antioxidant properties of TEL, previously observed only in pre-clinical models (20). Moreover, the beneficial effect shown by telmisartan may be explained by its multiple therapeutic characteristics. Indeed, TEL is a unique ARB with selective PPAR- $\gamma$-modulating activity which affects nitric oxide bioavailability thus leading to its anti-inflammatory, antioxidant and antiproliferative effects on vascular wall cells (21). Additionally, it has also been shown to play a role in lipid and glucose metabolism (22).

Cytokines, sensitive markers of tissue damage, are responsible for a negative inotropic effect in the failing human heart $(1,12)$ and in the pathophysiology of dilated cardiomyopathy (22). The increase in proinflammatory cytokines (IL-6) and oxidative stress markers (2) after EPI administration confirms that systemic inflammation/oxidative stress plays a central role in the cardiac damage induced by EPI. Indeed, significant correlations between cytokines/ROS levels and SR decline, observed in our previous study (8) and confirmed in the PLA arm of the present trial, suggest that an increase in inflammatory/ROS markers may be analogous to early myocardial cell dysfunction shown by TDI.

A pathogenetic hypothesis based on oxidative stress has gained the widest acceptance in the study of acute EPI-induced CTX. Its molecular basis is attributable to the one-electron redox cycling of the quinone moiety, which generates ROS in excess of limited cardiomyocyte antioxidant defenses (23). This cellular pathway results in severe oxidative stress and disruption of the mitochondrial energetic machinery, ultimately leading to cardiomyocyte apoptosis or necrosis (24). Indeed, a relationship was found between cytokine release and ROS increase in patients with dilated cardiomyopathy (25).

The observation that TEL is able to prevent such a number of potentially harmful effects induced by EPI, to which, however, its antineoplastic therapeutic efficacy is attributable, suggests that its administration may also compromise or weaken the antitumor efficacy of ANT. To date, however, this hypothesis is not supported by any data in the literature.

Regarding the use of TEL as a cardioprotective drug in our present trial, it is to be noted that very recently (July 2010), and long after the beginning of our study (end of 2008) and concomitantly with the publication of the first results (September 2010), a meta-analysis was published by Sipahi et al (26). The authors concluded that when the analysis was limited to TEL, the excess in new cancer (lung cancer) occurrence was of borderline significance $(\mathrm{p}=0.05)$ and that no statistically significant difference in cancer deaths was observed. Furthermore, the TEL dose used in the trials which were reviewed in the meta-analysis was $80 \mathrm{mg} /$ day, i.e., double the dose used in our trial. For these reasons we believe that the findings of Sipahi et al have no bearing on our study.

In conclusion, the present study augments the findings of our earlier research (14) which aimed to assess the cardioprotective effect of TEL only during the period of EPI administration. The present study subsequently demon- 
strated that the protection obtained with the AT1R blockade had a long-lasting effect, probably by ensuring a permanent (at least up to 12-month FU) defense against chronic or lateonset types of ANT-induced CTX. This finding is extremely important, since ANT-induced CTX persists for years with no clinical symptoms, whereas upon the development of overt heart failure, the prognosis becomes extremely poor, possibly even worse than that of ischemic or idiopathic dilated cardiomyopathy.

\section{Acknowledgements}

This study was partially funded by the AIRC (Associazione Italiana Ricerca per il Cancro), project no. 8679.

\section{References}

1. Escobar E, Rodriguez-Reyna TS, Arrieta O and Sotelo J: Angiotensin II, cell proliferation and angiogenesis regulator: biologic and therapeutic implications in cancer. Curr Vasc Pharmacol 2: 385-399, 2004.

2. Thompson KL, Rosenzweig BA, Zhang J, et al: Early alterations in heart gene expression profiles associated with doxorubicin cardiotoxicity in rats. Cancer Chemother Pharmacol 66: 303-314, 2010.

3. Funao K, Matsuyama M, Kawahito Y, et al: Telmisartan is a potent target for prevention and treatment in human prostate cancer. Oncol Rep 20: 295-300, 2008.

4. Paulides $\mathrm{M}$ and Wojnowski L: [Chemotherapeutics-induced heart failure]. Med Klin 102: 574-578, 2007 (In German).

5. Meldrum DR, Cleveland JC Jr, Cain BS, Meng X and Harken AH: Increased myocardial tumor necrosis factor-alpha in a crystalloid-perfused model of cardiac ischemia-reperfusion injury. Ann Thorac Surg 65: 439-443, 1998.

6. Kupatt C, Habazettl H, Goedecke A, et al: Tumor necrosis factor-alpha contributes to ischemia- and reperfusion-induced endothelial activation in isolated hearts. Circ Res 84: 392-400, 1999.

7. Mercuro G, Cadeddu C, Piras A, et al: Early epirubicin-induced myocardial dysfunction revealed by serial tissue Doppler echocardiography: correlation with inflammatory and oxidative stress markers. Oncologist 12: 1124-1133, 2007.

8. Mantovani G, Madeddu C, Cadeddu C, et al: Persistence, up to 18 months of follow-up, of epirubicin-induced myocardial dysfunction detected early by serial tissue Doppler echocardiography: correlation with inflammatory and oxidative stress markers. Oncologist 13: 1296-1305, 2008.

9. Toko H, Oka T, Zou Y, et al: Angiotensin II type 1a receptor mediates doxorubicin-induced cardiomyopathy. Hypertens Res 25: 597-603, 2002.
10. Soga M, Kamal FA, Watanabe K, et al: Effects of angiotensin II receptor blocker (candesartan) in daunorubicin-induced cardiomyopathic rats. Int J Cardiol 110: 378-385, 2006.

11. Oken MM, Creech RH, Tormey DC, et al: Toxicity and response criteria of the Eastern Cooperative Oncology Group. Am J Clin Oncol 5: 649-655, 1982.

12. Iqbal M, Dubey K, Anwer T, Ashish A and Pillai KK: Protective effects of telmisartan against acute doxorubicin-induced cardiotoxicity in rats. Pharmacol Rep 60: 382-390, 2008.

13. Ishiguro H, Ishiguro Y, Kubota Y and Uemura H: Regulation of prostate cancer cell growth and PSA expression by angiotensin II receptor blocker with peroxisome proliferator-activated receptor gamma ligand like action. Prostate 67: 924-932, 2007.

14. Cadeddu C, Piras A, Mantovani G, et al: Protective effects of the angiotensin II receptor blocker telmisartan on epirubicininduced inflammation, oxidative stress, and early ventricular impairment. Am Heart J 160: e481-e487, 2010.

15. Wenzel P, Schulz E, Oelze M, et al: AT1-receptor blockade by telmisartan upregulates GTP-cyclohydrolase I and protects eNOS in diabetic rats. Free Radic Biol Med 45: 619-626, 2008.

16. Stephen RL, Gustafsson MC, Jarvis M, et al: Activation of peroxisome proliferator-activated receptor delta stimulates the proliferation of human breast and prostate cancer cell lines. Cancer Res 64: 3162-3170, 2004.

17. Jensen BV, Skovsgaard T and Nielsen SL: Functional monitoring of anthracycline cardiotoxicity: a prospective, blinded, long-term observational study of outcome in 120 patients. Ann Oncol 13: 699-709, 2002.

18. Jalowy A, Schulz R and Heusch G: AT1 receptor blockade in experimental myocardial ischemia/reperfusion. Basic Res Cardiol 93 (Suppl 2): 85-91, 1998.

19. Ferreira AL, Matsubara LS and Matsubara BB: Anthracyclineinduced cardiotoxicity. Cardiovasc Hematol Agents Med Chem 6: 278-281, 2008.

20. Cianchetti S, del Fiorentino A, Colognato R, di Stefano R, Franzoni $\mathrm{F}$ and Pedrinelli R: Anti-inflammatory and antioxidant properties of telmisartan in cultured human umbilical vein endothelial cells. Atherosclerosis 198: 22-28, 2008.

21. Yamagishi S and Takeuchi M: Telmisartan is a promising cardiometabolic sartan due to its unique PPAR-gamma-inducing property. Med Hypotheses 64: 476-478, 2005.

22. Tuck ML: Angiotensin-receptor blocking agents and the peroxisome proliferator-activated receptor-gamma system. Curr Hypertens Rep 7: 240-243, 2005.

23. Minotti G, Menna P, Salvatorelli E, Cairo G and Gianni L: Anthracyclines: molecular advances and pharmacologic developments in antitumor activity and cardiotoxicity. Pharmacol Rev 56: 185-229, 2004.

24. Conklin KA: Coenzyme q10 for prevention of anthracyclineinduced cardiotoxicity. Integr Cancer Ther 4: 110-130, 2005.

25. Kaur K, Sharma AK, Dhingra S and Singal PK: Interplay of TNF-alpha and IL-10 in regulating oxidative stress in isolated adult cardiac myocytes. J Mol Cell Cardiol 41: 1023-1030, 2006.

26. Sipahi I, Debanne SM, Rowland DY, Simon DI and Fang JC: Angiotensin-receptor blockade and risk of cancer: meta-analysis of randomised controlled trials. Lancet Oncol 11: 627-636, 2010. 